\title{
Embryo culture goes back to the future
}

\section{David F. Albertini ${ }^{1}$}

Published online: 12 August 2020

(C) Springer Science+Business Media, LLC, part of Springer Nature 2020

"There is no such thing as unprejudiced observation. Every act of observation we make is biased. What we see, or otherwise sense, is a function of what we have seen or sensed in the past."

\section{Sir Peter Medawar}

Of the many insights Medawar provided to an embryonic cast of scientists and curiosity seekers in the 1960s was one prescient commentary made in an essay entitled "Is the scientific paper a fraud?" (From The Strange Case of the Spotted Mice and other classic essays on science, Oxford University Press, 1996, ISBN 0-19-286,193). The aforementioned quote from that essay introduced Medawar's overarching treatment of what was at that time the status quo for publishing novel findings that could or should influence advances in medicine. His skepticism was directed at a process we continue to engage in some 60 years later- a matter that has as much to do with reproductive medicine today as it did back then. For those of you unfamiliar with Sir Peter, bear in mind he was a Nobel laureate in 1960 for his ground breaking research in immunology and fittingly recognized as the prime mover of the concept of immunological recognition of pregnancy and the importance of host tolerance of the fetal allograft. This month we build on the subject of embryo culture from present to past and in the process take a decidedly skeptical (Medawarian) point of view given the congruence of practices today and evolving concerns over how human ARTs impacts the epigenetic health and lifestyle of offspring.

In some ways the story of human embryo culture from 1978 to today resembles more a matter a denouncement of the "Mother Nature" approach of yesteryear to an accommodation of workflow and commercial forces taking an ever greater hold on clinical ARTs in the moment. Sunde and

David F. Albertini

eicjarg@gmail.com

1 Bedford Research Foundation, Bedford, MA, USA colleagues took aim at the misguided efforts having to do with not simply the matter of media formulations, but single vs sequential, the high/low oxygen tension, Day 3 or day 5 and supplements or not (note how binary our options have been) among other manipulations, fostering a sense of confusion for the average practitioner looking out, supposedly, for the benefit of the patient [1]. Contemporaneously, we can add to the litany: to biopsy or not, to PGT-A or not, to deploy timelapse (morphokinetics) or not, to analyze medium for cell free DNA (or proteins, or metabolites), or not-all things embryo culture designed by and for our specialty with the expressed purpose of choosing the embryo most likely to yield a healthy child following transfer. Recall if you will that among the founding fathers and mothers of embryo culture for the mouse, recognizing and attempting to recapitulate the natural environment within which development occurs was a laudable and rewarding strategy, setting the stage for implementing a "chemically"defined medium [2]. Remarkably, (but not unexpected given the avoidance of older literature seen routinely in commercial ART platforms), it was patently obvious in the early 1970s that mitochondrial metabolism in the preimplantation mouse embryo was repressed or never active due to what might have been anticipated as the precarious consequences of generating free radicals [3]. And finally, at the crux of our embryo metabolism retrospective, was the matter of bioenergetics for a free-floating multicellular entity destined to establish two distinct cell lineages- could it be that our simplistic, but firmly entrenched, understanding of the pyruvate to glucose shift at the morula to blastocyst transition has led us astray? [4]. Surely, our trivialized preconceptions of preimplantation embryo metabolism would leave room for improvements if only the highly cited papers driving this field were to be challenged. This now seems to be the case.

Studying metabolism is a far more complicated enterprise in classical biochemistry than most of the attempts we have seen carried out with human embryos to date. Culture media metabolomics studies have added coarse data sets which, when subjected to "rigorous" bioinformatics analysis, have allowed our field to make informed guesses about what may 
or may not be happening during these critical transitions in early development. But now the tools are far more refined for teasing apart cell metabolism in mammalian embryos to ask just how conducive or damaging are our media formulations for survival and development. Here, the recent studies of Chi and colleagues on mouse embryos raise several intriguing questions about how to culture human embryos.

This tour de force set of experiments coupled the tracking isotope tagged carbon to determine the true fate and utility of glucose at a level of detail never before achieved for mammalian embryos. Their elegant studies show that glucose is entirely dispensable as an energy substrate during the morula to blastocyst transition [5]. Moreover, they convincingly demonstrate using metabolic pathway specific inhibitors that glucose is utilized not for bioenergetics driving development but for specifying trophectoderm cell fate, leaving determination of ICM lineages up to cell contact relationships and positional information. This work is a game changer! If established as translatable to human embryos, it is likely to alter our current mindset regarding embryo metabolism, culture components, and length of culture. For those who may have noticed during these past years of extended culture, the problems associated with oxidative stress have come into the limelight as efforts have been refocused by adding antioxidants to media. By the way, it was only 50 years ago that the importance of physiological anti-oxidants like reduced glutathione, was shown to be permissive for mouse development past the 2-cell stage [6].

Why re-examine out approach to embryo culture beyond the fact that fundamental aspects of what we perceived to be important may turn out to be irrelevant? For one thing, concerns over short or long term effects of culture on epigenetic modifications impacting offspring development have been proposed in mouse models where long term follow-up studies have been revealing and disconcerting at the same time [7]. Again, whether such a relationship between ex vivo exposures (embryo culture) translated to the human condition has been an open question. The paper by Elder and Menezo this month will go a long way in bringing this problem into focus for our readership (Epigenetic remodeling of chromatin in human ART: addressing deficiencies in culture media https://doi. org/10.1007/s10815-020-01884). Extending this line of reasoning beyond the realm of practical embryo culture concerns to how the environments we choose to sustain human embryos in impact offspring health continues to raise red flags taken seriously by only a handful of ART practitioners. If you had doubts about the connection between imprinting disorders in ART offspring, look no further than the recent paper by Hara-Isono and colleagues [8].

We leave our readership with a glimpse into the role of bias in our decision-making steps at the very heart of human ARTs. Consider for a moment the prevalence of literature that has appeared in the past few years on the importance of mitochondria for powering the extraordinary morphogenesis witnessed in the timelapse series of so many embryos over the same time frame. Is it no wonder that because ATP powers all cell movements, the dynamic behavior of blastomeres would be construed to be dependent upon oxidative phosphorylation, whether or not there were consequences due to free radical production and its accumulation from 5 days of culture, or more.

Finally, we welcome two new members to the Editorial Board this month, Cariol Lynn Curchoe and Akwasi Amaoko, and look forward to their contributions to the growth of JARG in the coming years.

Stay well.

\section{References}

1. Sunde A, Brison D, Dumoulin J, Harper J, Lundin K, Magli MC, et al. Time to take human embryo culture seriously. Hum Reprod. 2016;31(10):2174-82.

2. Biggers JD, Gwatkin RB, Brinster RL. Development of mouse embryos in organ cultures of fallopian tubes on a chemically defined medium. Nature. 1962;26(194):747-9.

3. Stern S, Biggers JD, Anderson E. Mitochondria and early development of the mouse. J Exp Zool. 1971;176(2):179-91.

4. Brison DR, Sturmey RG, Leese HJ. Metabolic heterogeneity during preimplantation development: the missing link? Hum Reprod Update. 2014;20(5):632-40.

5. Chi F, Sharpley MS, Nagaraj R, Roy SS, Banerjee U. Glycolysisindependent glucose metabolism distinguishes TE from ICM fate during mammalian embryogenesis. Dev Cell. 2020;53(1):9-26 e4.

6. Cholewa JA, Whitten WK. Development of two-cell mouse embryos in the absence of a fixed-nitrogen source. J Reprod Fertil. 1970;22(3):553-5.

7. Feuer SK, Donjacour A, Simbulan RK, Lin W, Liu X, Maltepe E, et al. Sexually dimorphic effect of in vitro fertilization (IVF) on adult mouse fat and liver metabolomes. Endocrinology. 2014;155(11): 4554-67.

8. Hara-Isono K, Matsubara K, Mikami M, Arima T, Ogata T, Fukami $\mathrm{M}$, et al. Assisted reproductive technology represents a possible risk factor for development of epimutation-mediated imprinting disorders for mothers aged $>/=30$ years. Clin Epigenetics. 2020;12(1):111.

Publisher's note Springer Nature remains neutral with regard to jurisdictional claims in published maps and institutional affiliations. 